Dulce María Clemente Guerrero, Armando Rosas González, Alberto Antonio-García, Noemí Cruz Martínez, Edith Ramos Velasco

\title{
Conceptualización de sistema acuapónico aplicando herramientas del diseño concurrente ${ }^{1}$
}

\author{
Conceptualization of aquaponic system applying \\ concurrent design tools
}

\section{Cómo citar:}

Clemente, D., Rosas, A., Antonio-García, A., Cruz, N. \& Ramos, E. (2020). Conceptualización de sistema acuapónico aplicando herramientas del diseño concurrente. Designia, 8(1), 17-49

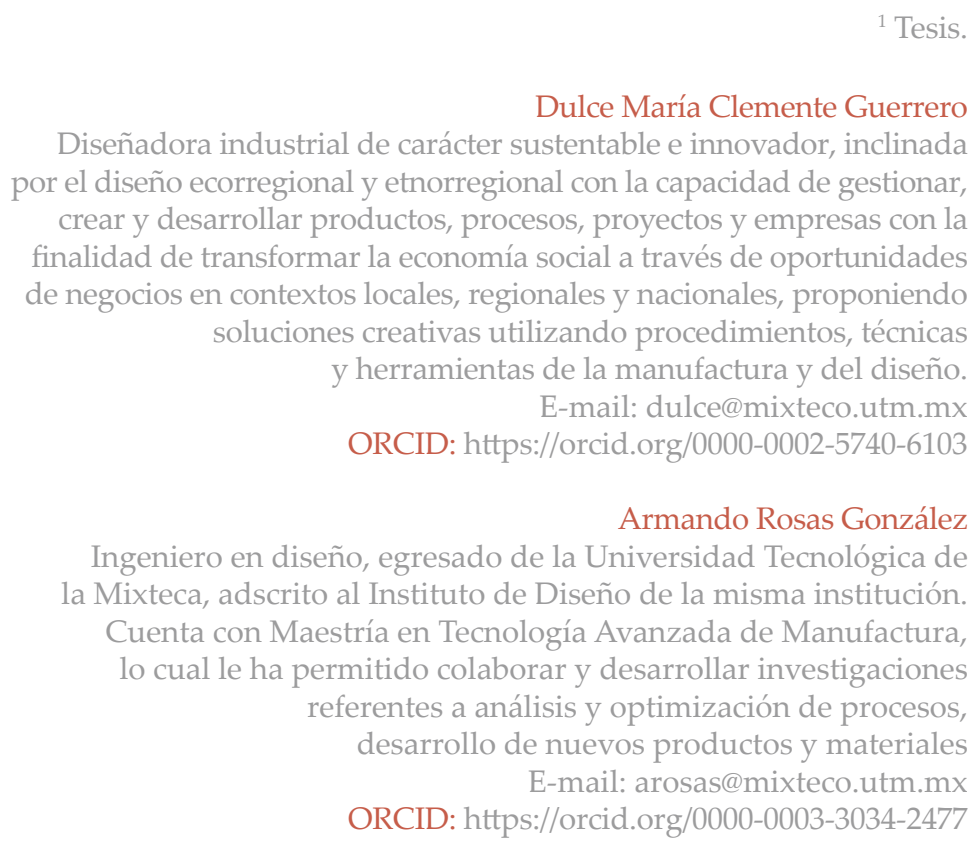

Palabras clave:

Acuaponía, despliegue de la función de la calidad, diseño asistido por computadora, ingeniería asistida por computadora, análisis de elemento finito, técnica de película de nutrientes.

Key words:

Aquaponics, quality function deployment, computer aided design, computer aided engineering, finite element analysis, nutrient film technique.

Recibido: 4/09/2019 Aceptado: 6/03/2020 
Alberto Antonio-García

Doctor en Ciencias en Ingeniería Mecánica en la sección de Estudios de Posgrado e Investigación del Instituto Politécnico Nacional (Esime-IPN). Su tesis sobre el cálculo de coeficientes rotodinámicos de chumaceras hidrodinámicas fue reconocida por el IIE y la CFE con el segundo lugar en los XXIII Certámenes Nacionales de Tesis

2005-2006, en la disciplina de generación de energía eléctrica.

E-mail: antoniog@mixteco.utm.mx

ORCID: https://orcid.org/0000-0002-4042-3740

Noemí Cruz Martínez Ingeniera en diseño egresada de la Universidad Tecnológica de la Mixteca, ejerce como diseñadora en áreas industriales, gráficas y arquitectónicas. E-mail: noemi_id@live.com.mx ORCID: https://orcid.org/0000-0002-3728-7557

Edith Ramos Velasco Ingeniera en diseño egresada de la Universidad Tecnológica de la Mixteca. Técnico del Departamento de Proyectos en la Universidad del Mar. E-mail: edith.ramos.utm@gmail.com ORCID: https://orcid.org/0000-0002-9782-1299

\section{Resumen:}

Con el paso del tiempo se han incrementado los desafíos relacionados con la producción y abastecimiento de alimentos, llegando a convertirse en un factor crítico para garantizar la seguridad alimentaria en todo el mundo. Esta situación ha favorecido la práctica de actividades sostenibles y el consumo de productos ecológicos. La acuaponía se constituye en ese sentido en un sistema alternativo para la producción de alimentos, en donde se integran la hidroponía (cultivo de plantas en agua sin suelo) y la acuicultura (cría de especies acuáticas). Sin embargo, la oferta de sistemas acuapónicos en México es limitada, y normalmente estos deben importarse del extranjero; por otra parte, la mayoría de las personas han implementado sistemas acuapónicos a nivel casero de manera improvisada, adaptando elementos prefabricados con falencias en su funcionamiento y un diseño poco atractivo. 
En este artículo de investigación se desarrolló a nivel conceptual el diseño de un sistema acuapónico (SA) para una escala de autoconsumo, el cual está destinado para hombres y mujeres de entre 24 y 35 años, pertenecientes a los niveles socioeconómicos A/B y C+ de la ciudad de Huajuapan de León, Oaxaca. Estas personas habitan en una región urbana árida, en donde existe una constante escasez de agua y se tienen pocas áreas agrícolas. Para el desarrollo del proyecto se aplicó la metodología propuesta por Riba y Molina (2006), conjuntamente con algunas herramientas del diseño concurrente para facilitar el trabajo interdisciplinario de profesionales que compartieron sus conocimientos en las áreas de mecánica de fluidos, antropometría, hidroponía y acuicultura.

El SA conceptualizado es un elemento que ocupa una superficie menor a dos metros cuadrados; su principal innovación radica en que integra áreas de cultivo verticales y horizontales, atributo que no posee ninguno de los sistemas acuapónicos existentes en el mercado y que permite un mayor aprovechamiento de la superficie de producción. En este podrán sembrarse hasta 64 plantas de 10 diferentes especies, combinando módulos de sustrato y contenedores con la técnica de película de nutrientes (NFT). Igualmente, también pueden cultivarse peces de tres especies distintas, en un acuario con capacidad para 300 litros de agua. De esta forma se conceptualizó un sistema que podría representar una alternativa ante la escasa oferta existente en México, buscando atender en su proyección las deficiencias de productos semejantes que actualmente se fabrican y comercializan.

\section{Abstract:}

As time goes by, challenges related to food production and supply have increased, becoming a critical factor in ensuring food security worldwide. This situation has favored the practice of sustainable activities and consumption of organic products. Aquaponics has become an alternative system for food production where hydroponics (growing plants in water without soil) and aquaculture (breeding of aquatic species) are integrated. However, supply of aquaponic systems in Mexico is limited, and usually they must be imported from abroad; also, most people have implemented aquaponic systems at home improvisedly, adapting prefabricated elements faulty in operation and with an unattractive design.

In this research paper a conceptual Aquaponic System (AS) design was developed for a self-consumption scale, intended for men and women from 24 to 35 years old, belonging to the socioeconomic levels A / B and C+ from Huajuapan de Leon, Oaxaca. These people live in an urban arid region where there is a constant water shortage and have few agricultural areas. To develop 
the project, the methodology proposed by Riba and Molina (2006) with some Design Concurring tools were jointly applied to facilitate interdisciplinary work of professionals, who shared their knowledge in fluid mechanics, anthropometry, hydroponics and aquaculture.

The conceptualized AS will occupy an area smaller than two square meters; the main innovation of the AS is that it integrates vertical and horizontal cultivation areas, an attribute that doesn't have any of the existing aquaponic systems, and it will allow a greater use of the production area. In this system, up to 64 plants of 10 different species can be planted, combining substrate modules and containers with Nutrient Film technique. In the projected AS, fish of three different species can also be grown in an aquarium with a capacity for 300 liters of water. Thus, a system was conceptualized to represent an alternative to the scarce supply of similar systems exist in Mexico; it's projection sought to address the deficiencies of similar products that are currently manufactured and sold.

\section{INTRODUCCIÓN}

La aceleración del crecimiento poblacional en las grandes urbes es un fenómeno que no ha cesado, y se estima que para el año 2050 el 80\% de los habitantes en la tierra residirán en zonas urbanas (Díaz, González, Sención y González, 2016). Asimismo, se han ido incrementando los desafíos relacionados con la producción y abastecimiento de alimentos, llegando a convertirse en un factor crítico para la seguridad alimentaria en todo el mundo. Usualmente, la mayoría de los alimentos se obtiene de sitios distantes a las zonas urbanas, lo cual incrementa su costo, demora su consumo y facilita su deterioro (FAO, 2003). A lo largo de la historia, diferentes sociedades han desarrollado estrategias para la producción orgánica y sustentable de diversos alimentos; entre ellas se encuentra la acuaponía, sistema que consiste en la integración de la hidroponía (cultivo de plantas en agua sin suelo) y la acuicultura (cría de especies acuáticas). En este sistema, las plantas cultivadas hidropónicamente obtienen nutrientes a partir de los desechos de alimentos no consumidos por los peces y de sus heces (Martínez, 2013; Gómez et al., 2015).

La acuaponía tiene sus raíces en la antigua China y Tailandia, donde los agricultores tenían sistemas para criar pollos y cerdos en jaulas que eran colocadas sobre un estanque donde cultivaban carpas y otras especies acuáticas. En Mesoamérica, los aztecas practicaron una forma propia de acuaponía mediante la crianza de peces junto a las cosechas: ellos construían islas artificiales conocidas como chinampas, donde sembraban maíz y otras plantas, y usaban los canales navegables que rodeaban estas islas para la 
crianza de peces, mientras que los desechos de estos que se sedimentaban en el fondo de los canales eran recuperados para fertilizar a las plantas (Pedroza y Criado, 2012). La mayoría de sistemas acuapónicos está compuesta por varios elementos esenciales (figura 1), tales como: 1) un tanque de peces o acuario; 2) un sistema de aireación, para proveer oxígeno a los peces; 3 ) un biofiltro, que es un contenedor que almacena materiales porosos como piedras, esponjas o biobolas; 4) un sumidero, donde el agua tiene salida a las tuberías; 5) una bomba de agua, para redirigirla desde el acuario de los peces hasta los cultivos hidropónicos y retornarla al origen en un circuito cerrado; 6) un área de cultivo, que puede incluir camas, canales o mesas (Colagrosso, 2014).

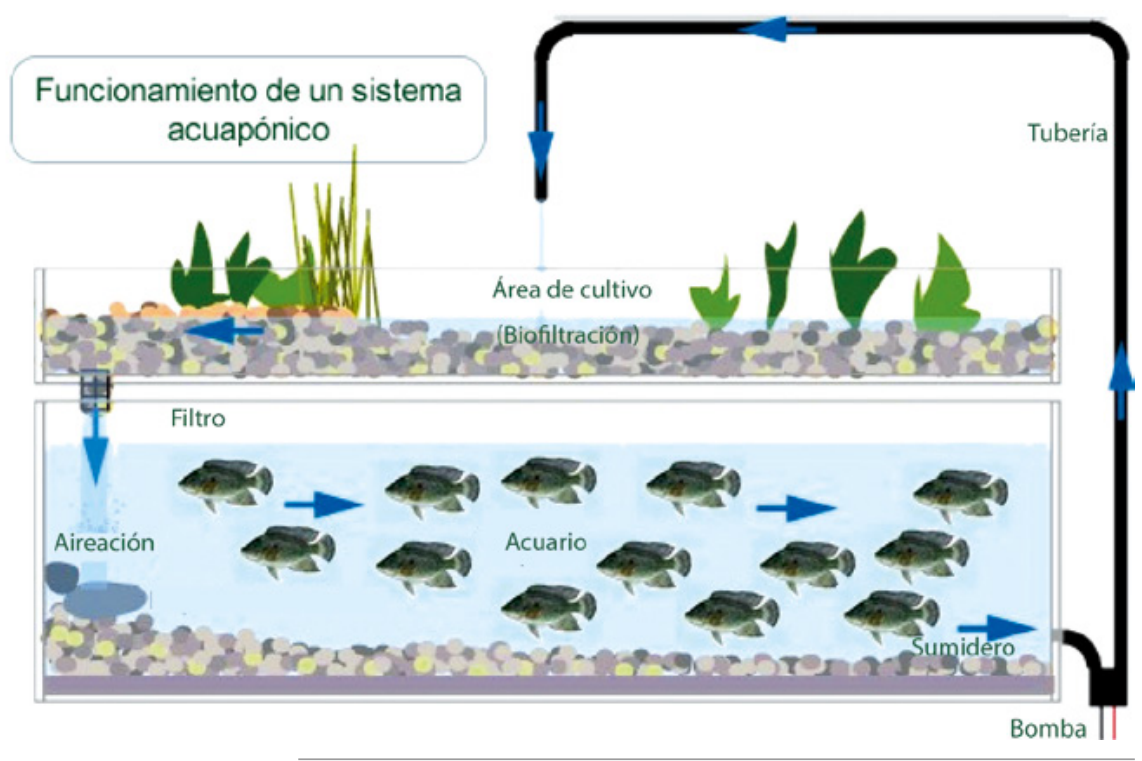

Figura 1. Elementos esenciales de un sistema acuapónico. Fuente: Elaboración propia, 2019.

Si bien existen muchas especies de peces y vegetales, no todas son aptas para su cultivo mediante acuaponía debido a diversos factores que afectan su desarrollo. Las especies de peces dulceacuícolas comestibles o de ornato y los vegetales de hojas verdes son los que mejor responden a estos sistemas (Ramírez, Sabogal, Jiménez y Hurtado, 2008). Por otro lado, son tres las principales técnicas de cultivo hidropónico usadas 
en acuaponía: 1) raíz o balsa flotante, hecha de contenedores plásticos que albergan planchas de poliestireno con vasos plásticos, los cuales contienen plantas cuyas raíces quedan inmersas en una solución nutritiva; 2) cama o lecho de sustrato, una técnica donde se tienen contenedores (cajones, bateas, etc.) llenos de un sustrato inerte que sirve de sostén a las plantas, mientras el agua circula constantemente entre un reservorio y los contenedores; 3) técnica de película de nutrientes (NFT), donde el agua circula por un conjunto de canales en una fina película y las plantas se colocan en recipientes de plástico suspendidos, los cuales contienen un sustrato inerte y perforaciones en el fondo para que las raíces queden inmersas en el agua (Rosillón et al., 2018).

El sistema NFT es la técnica más popularizada en hidroponía porque permite el aprovechamiento del espacio en lugares reducidos, altos rendimientos de producción por superficie, gran versatilidad y practicidad a la hora de su montaje; a su vez, el agua tiene una excelente oxigenación al estar en contacto con aire abundante dentro de las canaletas (Cutiño, Imeroni y Sanzano, 2018). Actualmente, en el mundo se desarrollan dos escalas diferentes de acuaponía: de mayor escala (comercial) y de menor escala, casera o de traspatio (autoconsumo).

Entre las ventajas que presenta la acuaponía con respecto a otras alternativas de producción agropecuaria sostenible se encuentran: 1) es factible su instalación en pequeños espacios; 2) la construcción de los sistemas acuapónicos es sencilla y pueden utilizarse contenedores reciclados; 3) la producción de cultivos es constante a lo largo del año; 4) se producen fácilmente especies acuáticas y plantas; 5) se tiene una mayor producción en comparación con la hidroponía, consumiéndose menos agua; 6) no se utilizan fungicidas o insecticidas; 7) este sistema puede implementarse a diferentes escalas (autoconsumo o comercial); 8) un SA puede ser operado perfectamente tanto por hombres como por mujeres, jóvenes o personas de la tercera edad (Martínez, R., 2013).

La Organización de las Naciones Unidas para la Alimentación y la Agricultura (FAO) ha señalado que en los últimos años la acuaponía se ha convertido en una opción para la alimentación a nivel mundial, como uno de los sistemas que conforman la agro-acuicultura integrada (AAI), a partir de la cual puede reducirse el consumo de agua en un 90\% en comparación con la agricultura tradicional (Rosillón et al., 2018). A pesar de que en México desde hace varios años se han realizado proyectos de sistemas experimentales y granjas comerciales de acuaponía, aún es una tecnología poco reconocida. En la república mexicana la mayoría de las personas que han implementado sistemas acuapónicos a nivel casero lo han hecho de manera improvisada, adaptando para esto elementos prefabricados que no han sido producidos en forma adecuada, con falencias en su funcionamiento y un diseño poco atractivo. Por otro lado, la oferta de sistemas acuapónicos en México es limitada, y en general estos deben importarse del extranjero, lo cual incrementa significativamente su precio (Gómez et al., 2015). 
La ciudad de Huajuapan de León se sitúa en el estado mexicano de Oaxaca, una de las localidades que más destacan debido a su importancia económica, social, política y cultural. De acuerdo con datos del Instituto Nacional de Estadística y Geografía (Inegi, 2015), hasta el año 2015 tuvo una población de 77547 habitantes, ubicándose como la quinta ciudad más poblada a nivel estatal. La principal actividad económica desarrollada es el comercio local y foráneo, seguido de la construcción, los servicios y las industrias manufactureras. Posee una superficie de $330.01 \mathrm{~km}^{2}$; tiene un clima semicálido húmedo, con lluvias en verano; el rango de temperaturas en la ciudad fluctúa entre 16 y $24^{\circ} \mathrm{C}$; su relieve se distingue por la presencia de numerosas montañas que forman parte de la Sierra Mixteca; la vegetación predominante es xerófita, compuesta principalmente por cactáceas, opuntias, matorrales y árboles característicos de las tierras áridas mexicanas. La superficie de Huajuapan de León está cubierta principalmente por cambisol cálcico, que es un suelo esencialmente agrícola, pero la constante escasez de agua en la localidad impide el desarrollo de la agricultura más allá de pequeñas huertas de subsistencia. La mayoría de los alimentos que adquieren los habitantes de Huajuapan de León provienen de localidades y estados cercanos (Instituto Nacional para el Federalismo y el Desarrollo Nacional-Inafed, 2020).

Debido a lo anterior, un grupo de investigación del Instituto de Diseño de la Universidad Tecnológica de la Mixteca desarrolló este proyecto, con el objetivo de diseñar a nivel conceptual un SA para una producción de autoconsumo, con áreas de cultivo verticales y horizontales, a partir de las necesidades que fueron identificadas con usuarios potenciales de la ciudad. En correspondencia con las condiciones ambientales y de operación que fueron consideradas, el diseño conceptual del SA incluyó la definición de los elementos del sistema acuícola (especies de peces, acuario, aireación), del sistema de conducción, control y regulación hídrica (instalación hidráulica, filtros), del sistema de cultivo (especies vegetales, técnicas de cultivo, sistema de iluminación) y de los elementos de diseño (formas, dimensiones, materiales, métodos de ensamble, procesos de manufactura). También se obtuvo el modelo 3D virtual del SA usando el software Solidworks ${ }^{\circledR}$, y se hizo un análisis comparativo de algunas de sus características con respecto a las de un producto de referencia. De esta manera, se generó el diseño conceptual de un sistema que podría representar una alternativa funcional y ornamental, ante la escasa y deficiente oferta de productos similares que existe en México. Además, con este proyecto se pretende resaltar la importancia que tiene la aplicación de herramientas del diseño concurrente y el trabajo interdisciplinario para el desarrollo de productos. 


\section{METODOLOGÍA}

Se utilizó la metodología propuesta por Riba y Molina (2006) para satisfacer los requerimientos formales y funcionales del proyecto. Esta metodología permitió la inclusión de distintos enfoques para el diseño de un producto (entorno de mercado, usuarios, diseñador, expertos en áreas específicas, etc.) y de herramientas como el despliegue de la función de calidad (QFD, en inglés; DFC, en español), diseño asistido por computadora (CAD) e ingeniería asistida por computadora (CAE). A continuación se presentan las etapas empleadas en esta investigación:

Etapa 1. Ideación: Se establecieron los requerimientos de diseño del proyecto, con base en los siguientes aspectos: 1) identificación y características de los usuarios; 2) revisión bibliográfica para conocer los atributos funcionales y formales más importantes en los sistemas acuapónicos; 3) identificación y caracterización de sistemas acuapónicos; 4) caracterización antropométrica de los usuarios potenciales; 5) entrevistas a especialistas que se ocupan del diseño y fabricación de sistemas hidropónicos y acuícolas; 6) desarrollo de la primera fase del DFC para la jerarquización de las necesidades identificadas y para definir los requerimientos de diseño.

Etapa 2. Desarrollo conceptual y básico: Se eligió la propuesta del sistema acuapónico por desarrollar, considerando los siguientes aspectos: 1) definición de la arquitectura del sistema; 2) revisión de pautas para el diseño de sistemas hidropónicos y acuícolas; 3) cálculo del caudal de agua; 4) determinación de características específicas para los elementos del SA; 5) generación de conceptos a través de matriz morfológica; 6) elaboración de bocetos; 7) selección de la propuesta mediante matriz de Pugh.

Etapa 3. Desarrollo avanzado: Se obtuvieron las especificaciones técnicas y la propuesta de diseño final del SA, a partir de los siguientes aspectos: 1) modelo 3D virtual de la propuesta seleccionada ocupando el software Solidworks $\left.{ }^{\circledR} ; 2\right)$ comprobación de la resistencia mecánica de algunos elementos del SA conceptualizado mediante el análisis de elemento finito (AEF); 3) planos constructivos.

Etapa 4. Evaluación: Se realizó una evaluación comparativa del SA desarrollado frente a un producto de referencia, contrastando los requerimientos de diseño establecidos en la primera fase del DFC. 


\section{RESULTADOS}

\subsection{Etapa 1. Ideación}

En esta etapa, el grupo de investigación conformado por ingenieros en diseño, ambientales y mecánicos, además de diseñadores industriales, comenzó por definir el perfil del usuario para el cual se diseñaría el sistema acuapónico. Se analizaron diferentes investigaciones para generar perfiles de consumidores de productos orgánicos y ecológicos en México, encontrándose que, a nivel nacional, el consumo de estos productos se presenta en jóvenes y adultos jóvenes, hombres y mujeres, entre los 24 y los 35 años, con estudios de nivel superior, pertenecientes a los niveles socioeconómicos A/B (nivel alto) y C+ (nivel medio alto). En dichos estudios, las mujeres con nivel socioeconómico medio alto son quienes tienen una mayor disposición de compra de productos orgánicos y ecológicos; estas mujeres pertenecen comúnmente a familias pequeñas (de cuatro o cinco miembros) con niños pequeños, $\mathrm{y}$ son personas que invierten la mayoría de su dinero en alimentos. Se halló que el consumo de productos para este perfil de usuarios está motivado por múltiples intereses e involucra distintas dimensiones de su vida cotidiana; en ese sentido, su alimentación se relaciona estrechamente con su salud y la búsqueda de sustentabilidad (Ramírez, 2007; Torres, 2014; Díaz, Pérez y Hernández, 2015). Estas personas son un tipo de consumidores que en mercadotecnia son denominados idealistas, quienes pagan precios premium para mejorar su calidad de vida, invierten en su alimentación y adquieren productos orgánicos y ecológicos (Gómez, 2005).

Se determinó entonces que los usuarios para quienes se diseñaría el SA son hombres y mujeres entre 24 y 35 años, pertenecientes a los niveles socioeconómicos $\mathrm{A} / \mathrm{B}$ y $\mathrm{C}+\mathrm{de}$ la ciudad de Huajuapan de León, debido a que en esta localidad se tienen condiciones ambientales que hacen factible el cultivo de vegetales y peces mediante la acuaponía, lo cual representaría una oportunidad adicional para que los habitantes puedan obtener algunos de sus alimentos. Aunado a lo anterior, allí mismo se localiza la institución en donde se desarrolló el proyecto.

Con el objetivo de obtener información para la proyección del SA se aplicaron encuestas a una muestra de habitantes de la ciudad de Huajuapan de León que pertenecen al perfil del usuario definido. El tamaño de la muestra (72 personas) fue calculado mediante un muestreo probabilístico estratificado, con base en los datos poblacionales municipales y socioeconómicos estatales brindados por el Inegi (2015) y la Amai (Asociación Mexicana de Agencias de Investigación de Mercado, 2016). 
Las encuestas estuvieron compuestas por diez preguntas, y a partir de sus resultados se obtuvieron las siguientes conclusiones: 1) el 90\% de las personas afirmó estar interesado en el cultivo de plantas; 2) el 58\% de los encuestados mencionó estar casado y con hijos y el 33\% declaró estar soltero; 3) la mayor parte de los personas tiene un nivel de escolaridad de licenciatura (32 personas) y de especialidad (21 personas); 4 ) los encuestados dijeron que están principalmente interesados en cultivar lechuga, fresa, tomate, hierbabuena, cilantro, espinaca, chile, orégano, acelgas y perejil; 5) estas personas estarían interesadas en adquirir un SA, debido a que es una opción sustentable para obtener sus alimentos, porque están interesados en el cuidado del medio ambiente, les gusta cultivar vegetales y podrían reducir los gastos en su alimentación; 6) la mayoría de los encuestados dijo que tendría disponibles en promedio $4 \mathrm{~m}^{2}$ para colocar el SA; 7) la mayoría comentó que les gustaría ubicar el SA en la sala o en el patio; 8) para los encuestados, las características de un SA que consideran importantes son, en orden de prioridad: que sea para una producción de autoconsumo; que optimice espacios; que sea resistente mecánicamente; que en el mismo se puedan cultivar alrededor de diez especies diferentes de plantas; que permita guardar instrumentos y alimento para los peces; que sea estético, fácil de limpiar, fácil de instalar; que combine áreas de cultivo verticales y horizontales.

Enseguida, el grupo de investigación hizo un análisis de las características de sistemas acuapónicos que existen en el mercado (ver figura 2). Se encontró que todos los productos no se fabrican en México, pero pueden adquirirse mediante pedidos específicos. Del estudio comparativo efectuado se concluyó que: 1) son SA de menor escala (autoconsumo); 2) la técnica de cultivo con sustrato es la más utilizada en dichos sistemas, debido a su fácil manejo, mínimo mantenimiento, y por adaptarse a diferentes formas de contenedores; 3 ) algunos de los SA examinados presentan una construcción "hágalo usted mismo" (productos Paivert y Malthus), lo cual dificulta su instalación ya que el usuario debe configurar varias piezas de acuerdo al espacio con que cuenta; 4) todos los sistemas analizados incorporan áreas de cultivo de vegetales en una sola dirección (solo horizontales o solo verticales); 5) las áreas de cultivo de peces y vegetales son reducidas; 6) son difíciles de trasladar; 7) la mayoría muestra un aspecto tosco que los hace poco atractivos.

Después se entrevistó a los expertos en hidroponía y acuaponía del equipo de investigación, para conocer algunas necesidades adicionales del SA, determinándose las siguientes: 1) es recomendable que la aireación del SA para una producción de autoconsumo sea por gravedad (aireador de cascada); 2) la iluminación para las áreas de cultivo del SA debe tener una radiación fotosintéticamente activa (PAR) entre los 400 y $700 \mathrm{~W} / \mathrm{m}^{2}$ y una densidad de flujo de fotones sintéticos (PPFD) superior a 500 $\mu \mathrm{mol} / \mathrm{m}^{2} \mathrm{~s}$ en espacios interiores; 3 ) el SA debe contar con un sistema de recirculación 
que mantenga el flujo continuo del agua; 4) debe tener filtros para retener la materia orgánica; 5) se recomienda organizar las áreas de cultivo acorde a las especies vegetales a plantar; 6) es necesario calcular el caudal que circularía por el SA para garantizar su funcionamiento óptimo. Posteriormente, el grupo de investigación estableció un esquema general del SA proyectado para una producción de autoconsumo, con la finalidad de organizar sus elementos principales.

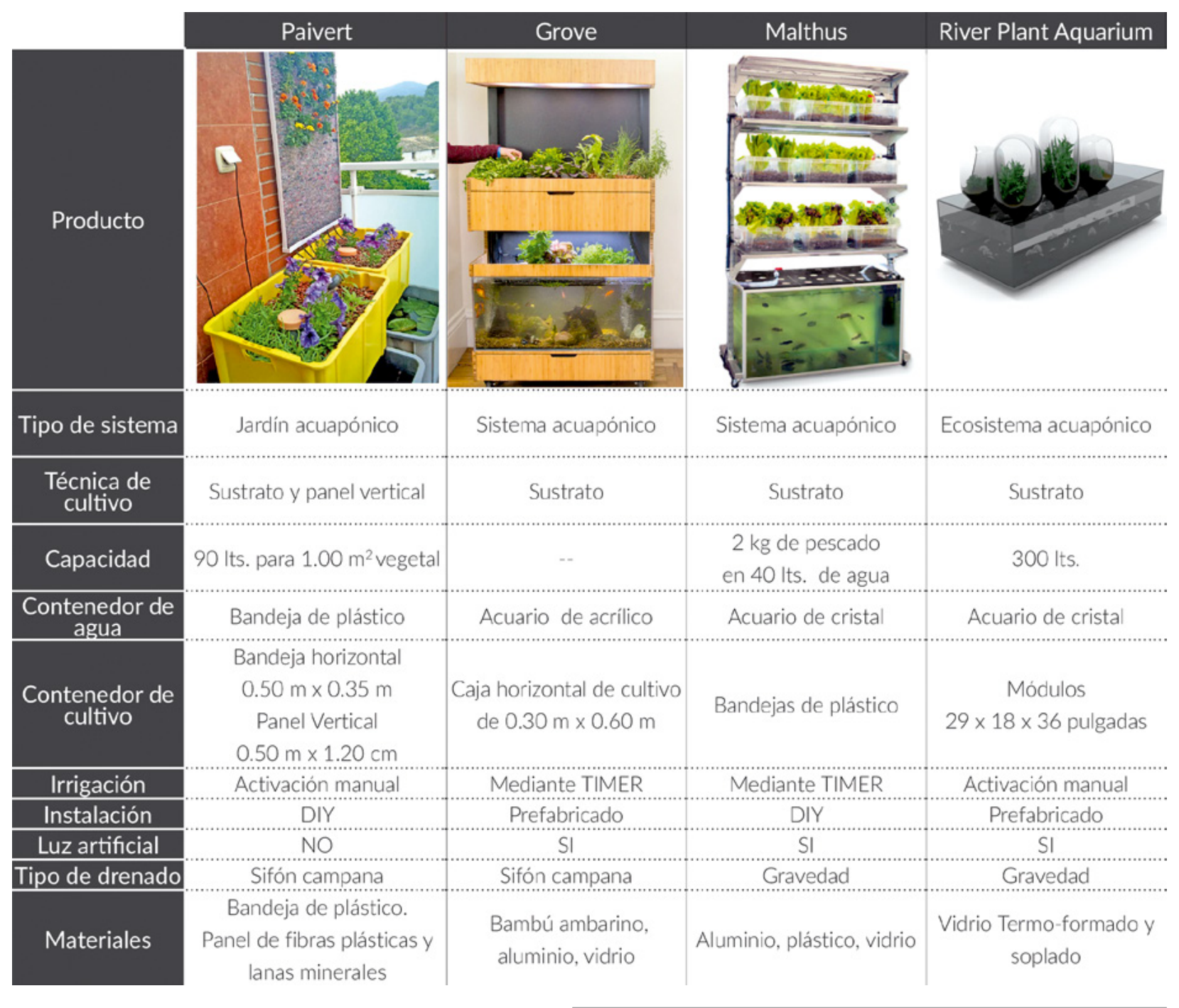

Figura 2. Tabla comparativa de SA comercializados. Fuente: Elaboración propia, 2019. 
Se determinaron cuatro elementos: 1) sistema acuícola; 2) sistema de conducción, control y regulación hídrica; 3 ) sistema de cultivo; 4) elementos de diseño (ver figura 3). Cada uno de los elementos anteriores incluye subelementos que intervinieron en la definición de los requerimientos de diseño del SA. Los elementos de diseño que se incluyeron están relacionados con pautas de antropometría, conocimientos de métodos de ensamble, procesos de manufactura y materiales que se necesitaron para definir la forma, dimensiones e instalación del sistema.

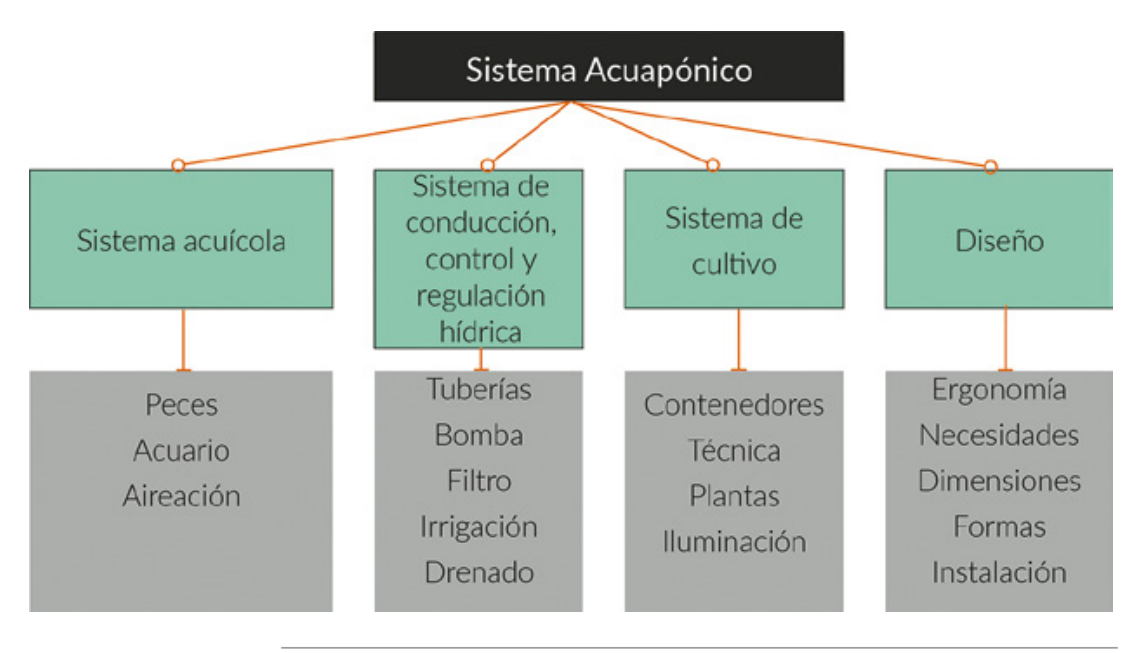

Figura 3. Elementos principales del SA.

Fuente: Elaboración propia, 2019.

Enseguida, el equipo de investigación desarrolló la primera matriz del despliegue de la función de la calidad (figura 4), donde se determinaron los requerimientos de diseño para cada una de las necesidades identificadas, tomando en consideración la información obtenida de las encuestas con los usuarios, del análisis comparativo de sistemas acuapónicos similares, y de las consultas y entrevistas con expertos. En la matriz puede observarse que se estableció un grado de relación entre las necesidades y requerimientos de diseño, que osciló en nueve unidades para aquellos elementos que guardaron una relación muy cercana, en tres unidades para aquellos que tuvieron una relación media, una unidad para los que tuvieron una relación baja y cero unidades para los aspectos que no tuvieron relación. Además, se determinó un grado de importancia para cada necesidad y requerimiento de diseño del SA. 


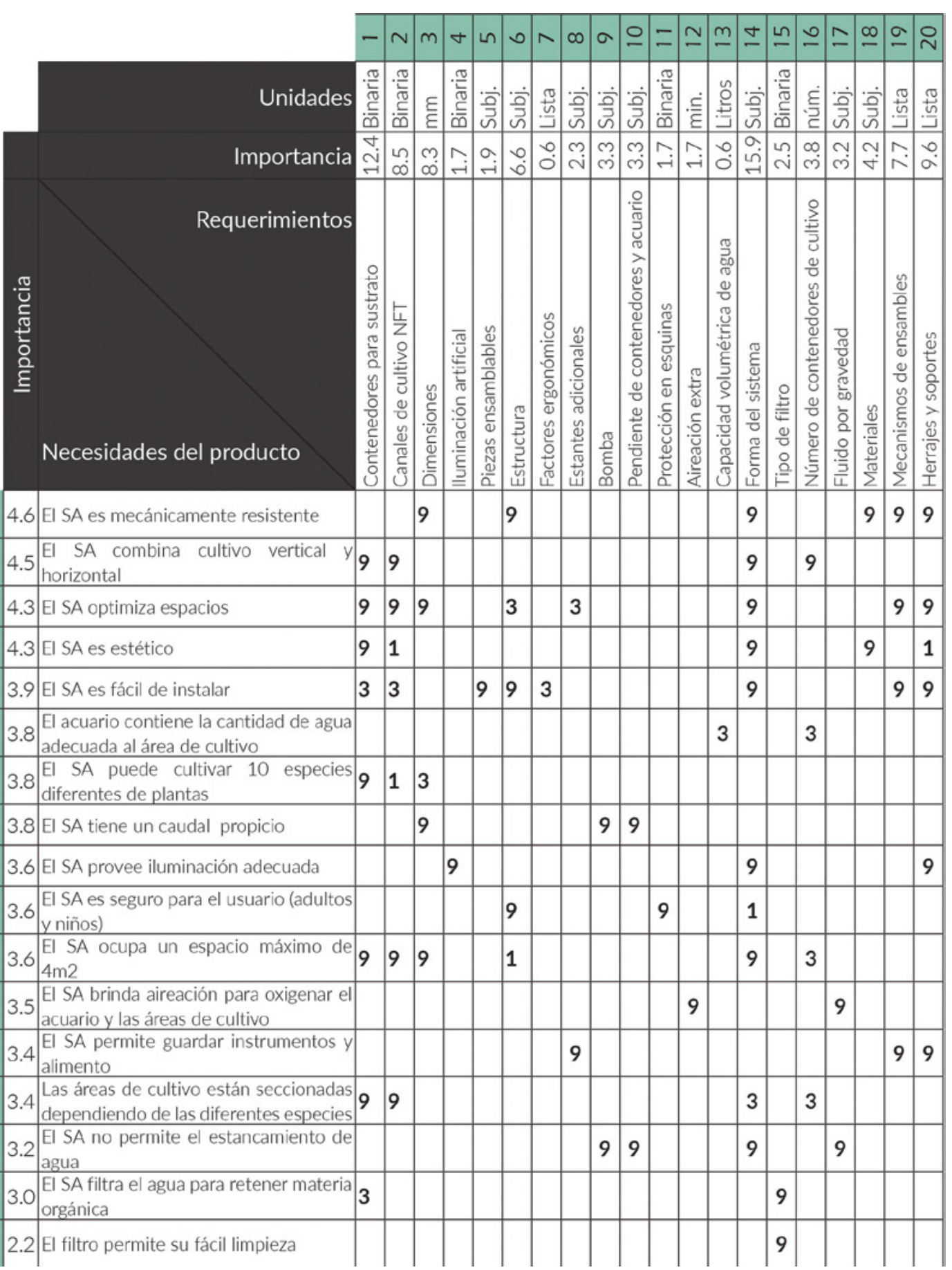




\subsection{Etapa 2. Desarrollo conceptual y básico}

En esta etapa se definió la arquitectura del SA (figura 5). El esquema incluye los elementos indispensables para su adecuado funcionamiento, además de que se indica el recorrido de la red hídrica con línea continua, las áreas de cultivo se representaron con línea discontinua y las uniones de algunos elementos se indicaron con puntos continuos. En el diagrama se representa el recorrido del agua en el SA, de acuerdo con el siguiente orden: se inicia con la entrada del agua al acuario, luego pasa por un filtro para después ser dirigida a las áreas de cultivo verticales y horizontales mediante una bomba, y finaliza su circulación en un segundo filtro para posteriormente reiniciar el ciclo. Se consideró que los filtros se tratarían como elementos independientes de la estructura metálica; en cambio, sobre ella se deberán colocar el acuario, la instalación hidráulica, el sistema de iluminación, el estante para materiales y las áreas de cultivo de vegetales. Posteriormente, se elaboraron alternativas para el diseño del SA mediante bocetos que incluyeron la descripción de sus atributos funcionales y formales. Las propuestas se obtuvieron a través de la aplicación de la matriz morfológica de Pugh.

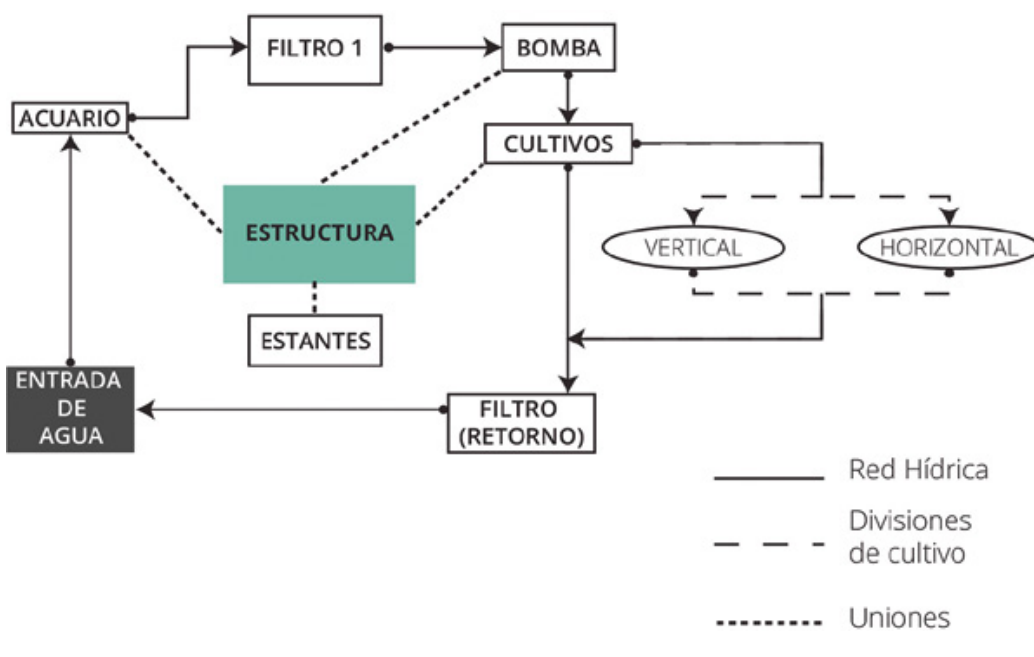

Figura 5. Arquitectura del SA. Fuente: Elaboración propia, 2019.

Se dividió la estructura del SA en siete elementos principales (figura 6), y para cada uno de ellos se definieron por lo menos dos variantes que sirvieron para efectuar distintas combinaciones y producir diferentes conceptos de diseño. 


\begin{tabular}{|c|c|c|c|c|c|c|}
\hline $\begin{array}{l}\text { Contenedores } \\
\text { vertical/horiz. }\end{array}$ & $\begin{array}{c}\text { Canales } \\
\text { vertical/horiz. } \\
\text { NFT }\end{array}$ & Acuario & $\begin{array}{c}\text { Estantes } \\
\text { adicionales }\end{array}$ & Filtro & Estructura & Aireación \\
\hline En torres & $\begin{array}{c}\text { Bandeja con } \\
\text { canales incluidos }\end{array}$ & Circular & Deslizables & Comercial & Personalizable & Caidas de agua \\
\hline Panel vertical & $\begin{array}{c}\text { Canales } \\
\text { independientes }\end{array}$ & Escalonado & Modular & Criba & Integral & $\begin{array}{l}\text { Cortina de } \\
\text { burbujas }\end{array}$ \\
\hline Maceta (módulo) & & $\begin{array}{c}\text { Bajo y extendido } \\
\text { a lo horizontal }\end{array}$ & & & Compacto & \\
\hline
\end{tabular}

Figura 6. Matriz de Pugh aplicada en el proyecto.

Fuente: Elaboración propia, 2019.

\subsubsection{Sistema acuapónico}

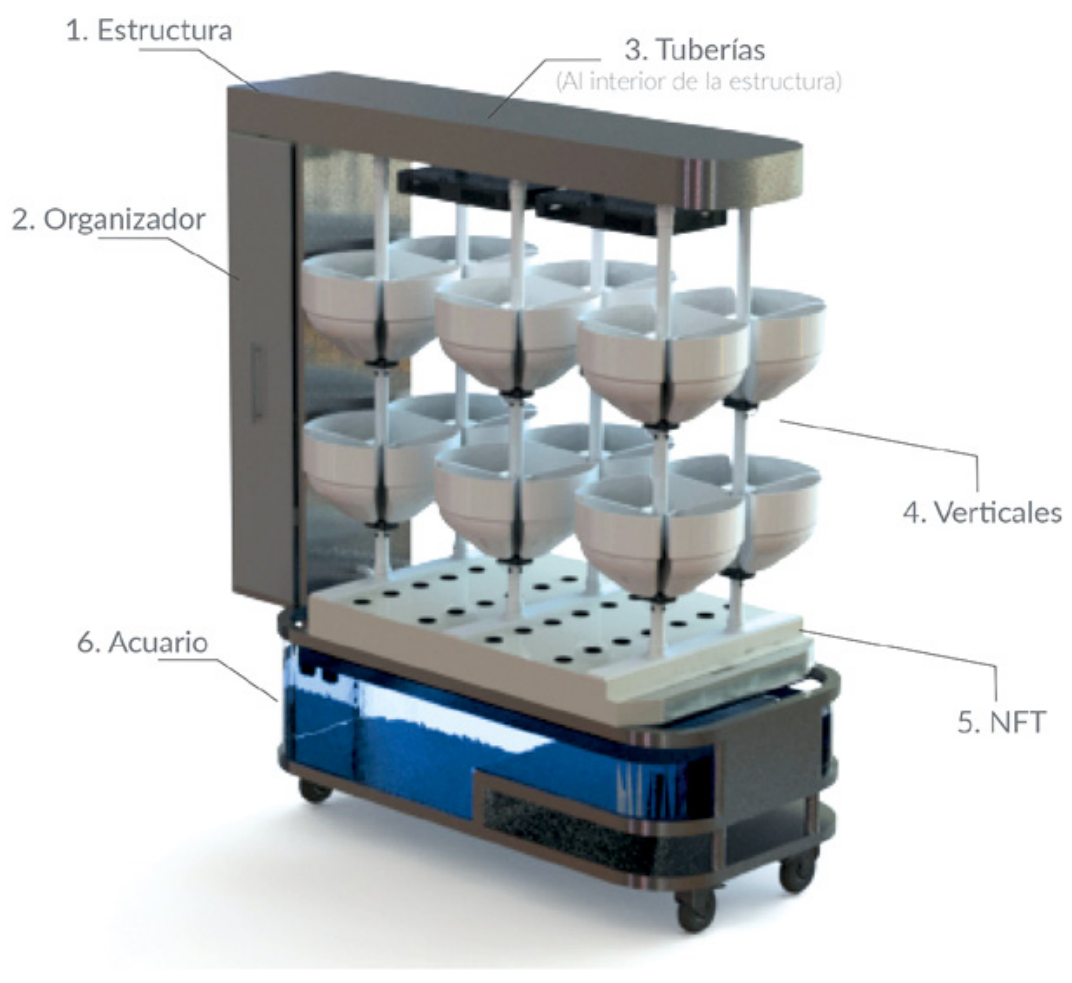

Figura 7. Vista general del SA. Fuente: Elaboración propia, 2019.

La propuesta seleccionada del SA se caracteriza por ser integral, en ella se buscó generar un equilibrio funcional y estético en el conjunto de sus partes (figura 7). Cuenta con un diseño contemporáneo atractivo que puede adaptarse a diferentes 
espacios interiores y exteriores, ocupando un volumen de $1.85 \times 0.80 \times 2.03 \mathrm{~m}^{3}$. En su estructura se incluyen diversos materiales como acero, vidrio y polímeros (figuras 8 y 9).

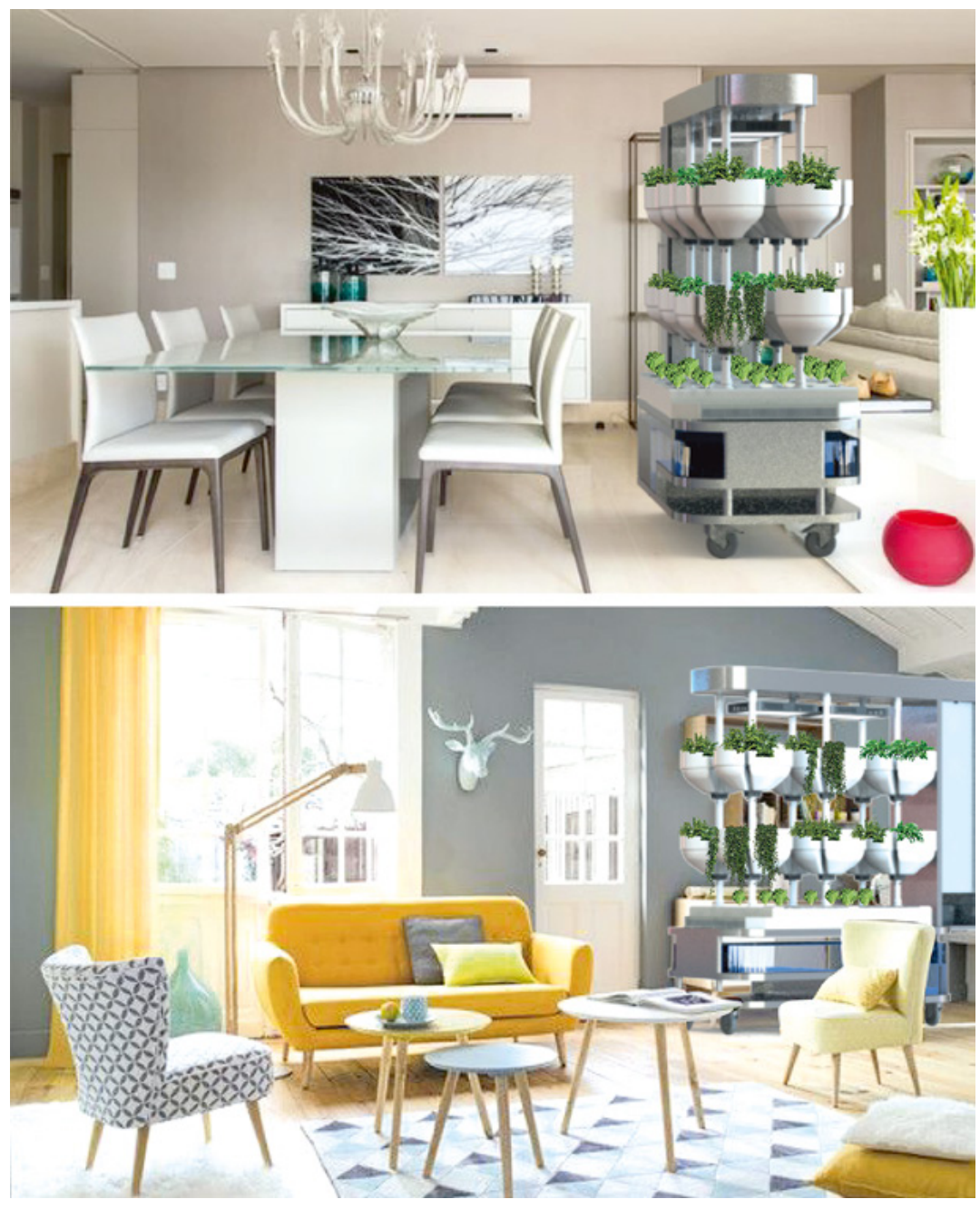

Figura 8. Vista del SA en un comedor. Figura 9. Vista del SA en una sala. Fuente: Elaboración propia, 2019. 
El SA incluye un acuario de baja altura, que se extiende en el plano horizontal para brindar un mayor espacio de movilidad a los peces, evitando que se estresen; además, cuenta con una geometría rectangular y esquinas redondeadas para impedir que los usuarios se lastimen. El acuario tiene una capacidad para $300 \mathrm{~L}$ de agua, presenta dos niveles con una diferencia de $0.15 \mathrm{~m}$ entre sus alturas, y tiene dos puntos de irrigación ubicados en sus extremos. En el lado izquierdo se ubica la tubería de succión para la bomba, y en el derecho se encuentra el punto de retorno del agua al acuario. El primer filtro se encuentra adjunto a la bomba de agua, para evitar que la materia orgánica de tamaño considerable sea enviada al área de los cultivos vegetales. La bomba impulsa el agua hasta la parte superior de la estructura, en donde se encuentra un punto de distribución que la reparte por seis tubos verticales, los cuales irrigan a los 36 contenedores con sustrato y a las 28 canastillas NFT.

Los contenedores de sustrato se distribuyen en torno a una matriz circular con respecto a cada tubo vertical; el área de cultivo NFT soporta a los tubos verticales y se localiza por encima del acuario; las canastillas NFT se encuentran irrigadas por 7 canales. El SA permite el cultivo de 10 especies de plantas y de 3 especies de peces, con un área vegetal de $2.30 \mathrm{~m}^{2}$, donde se podrán sembrar hasta 64 plantas y se podrán desarrollar de 5.75 a $11.50 \mathrm{~kg}$ de pescado. El sistema incluye además un estante para materiales, un montaje de iluminación para espacios cerrados y llantas para poder trasladarlo.

En la definición de los atributos formales del SA se consideraron las siguientes pautas de diseño sensorial brindadas por Bedolla (2002), enfocadas en la población objeto especificada: 1) implementar formas que conduzcan al usuario a la experimentación de asociaciones y sensaciones positivas inmediatas: depuración de forma, armonía, equilibrio; 2) usar colores que permitan una correcta percepción visual en diversas condiciones de luz; emplear colores con alto valor lumínico y baja saturación; utilizar monocromía y bicromía; 3) aplicar un estilo contemporáneo de diseño; 4) comunicar un concepto de calidad y seguridad utilizando colores de baja luminosidad, formas regulares, finos espesores y textura lisa; 5) generar una frescura moderada como respuesta a los estados emocionales de sobreactivación (agresividad, ira).

Para garantizar que los usuarios tengan un óptimo manejo de los elementos del SA, se tomaron en cuenta los ángulos de visión recomendados por Mondelo, Bombardo, Busquets y Torada (2004) y el percentil 50 de mujeres adultas, correspondiente a la estatura de $1.57 \mathrm{~m}$, altura de codo flexionado de $0.96 \mathrm{~m}$, alcance máximo vertical de $1.89 \mathrm{~m}$, alcance frontal de brazo de $0.68 \mathrm{~m}$ y altura de ojos de $1.45 \mathrm{~m}$; datos retomados del estudio hecho por Ávila, Prado y González (2007). 


\subsubsection{Especies vegetales}

Las plantas se distribuirán en módulos de sustrato (áreas de cultivo verticales) y contenedores horizontales con la técnica de película de nutrientes (ver figura 10). Para la determinación de las diez especies vegetales a cultivar en el SA se consideraron las opiniones expresadas en las encuestas por los usuarios y las recomendaciones dadas por el experto en hidroponía. También se revisaron las pautas brindadas por la FAO (2014) y por Martorell (2017), concernientes a método de cultivo, espacio individual de desarrollo, profundidad de raíces, humedad, y niveles relativos de luz y sombra que necesita cada planta.

\begin{tabular}{|c|c|c|c|c|c|c|}
\hline Especie & $\begin{array}{l}\text { Distancia } \\
\text { entre plantas } \\
(\mathrm{cm})\end{array}$ & $\begin{array}{l}\text { Altura } \\
(\mathrm{cm})\end{array}$ & $\begin{array}{c}\text { Profundidad del } \\
\text { contenedor } \\
(\mathrm{cm})\end{array}$ & Luz & Humedad & $\begin{array}{l}\text { Método de } \\
\text { cultivo }\end{array}$ \\
\hline $\begin{array}{l}\text { Lechuga } \\
\text { (Lactuca sativa) }\end{array}$ & $20-25$ & $20-30$ & 10 & Directa & Abundante & NFT \\
\hline $\begin{array}{l}\text { Tomate } \\
\text { (Solanum } \\
\text { lycopersicum) }\end{array}$ & 30 & -- & 30 & Indirecta & Abundante & Sustrato \\
\hline $\begin{array}{l}\text { Fresa } \\
\text { (Fragaria) }\end{array}$ & $15-20$ & 20 & 20 & Indirecta & Moderado & Sustrato \\
\hline $\begin{array}{l}\text { Cilantro } \\
\text { (Coriandrum } \\
\text { sativum) }\end{array}$ & 15 & 30 & 10 & Directa & Moderado & Sustrato \\
\hline $\begin{array}{l}\text { Acelgas } \\
\text { (Beta vulgaris var. } \\
\text { cicla) }\end{array}$ & 25 & 18 & 20 & Directa & Moderado & $\begin{array}{c}\text { NFT } \\
\text { ó Sustrato }\end{array}$ \\
\hline $\begin{array}{l}\text { Chile } \\
\text { (Capsicum } \\
\text { annuum) }\end{array}$ & 25 & $30-40$ & 30 & Indirecta & Moderado & Sustrato \\
\hline $\begin{array}{l}\text { Perejil } \\
\text { (Petroselinum } \\
\text { crispum) }\end{array}$ & 15 & 20 & 20 & Directa & Abundante & Sustrato \\
\hline $\begin{array}{l}\text { Orégano } \\
\text { (Origanum vulgare) }\end{array}$ & 15 & 15 & 25 & Directa & Moderado & $\begin{array}{c}\text { NFT } \\
\text { ó Sustrato }\end{array}$ \\
\hline $\begin{array}{l}\text { Hierbabuena } \\
\text { (Mentha spicata) }\end{array}$ & 10 & 20 & 10 & Indirecta & Abundante & Sustrato \\
\hline $\begin{array}{l}\text { Espinaca } \\
\text { (Spinacia oleracea) }\end{array}$ & 15 & 20 & 10 & Directa & Moderado & $\begin{array}{c}\text { NFT } \\
\text { ó Sustrato }\end{array}$ \\
\hline
\end{tabular}

Figura 10. Especies vegetales cultivables en el SA. 


\subsubsection{Especies de peces}

Posteriormente, el equipo de investigación estableció las especies de animales acuáticos que podrían cultivarse en el SA, tomando como referencia las recomendaciones dadas por la FAO (2014) y los expertos participantes. En la figura 11 se enlistan los peces que fueron elegidos, considerando aquellos que podrían consumirse como alimento y que lograrían coexistir bajo condiciones similares.

\begin{tabular}{c|c:c}
\hline Especie & Tipo & Observación \\
\hline Tilapia (Oreochromis) & Comestible & Temperatura: $27-30^{\circ} \mathrm{C}$, \\
\hline Carpa (Cyprinus carpio) & Comestible & Bemperatura: $25-30^{\circ} \mathrm{C}$, \\
\hline Ovoviviparos & Ornamental & Temperatura: $20^{\circ} \mathrm{C}$, \\
\hline
\end{tabular}

Figura 11. Especies de peces cultivables en el SA. Fuente: Elaboración propia, 2019.

\subsubsection{Cálculo de la capacidad de alimentación}

Conforme a las pautas brindadas por la FAO (2014), se estimó que el acuario debe tener una capacidad de $300 \mathrm{~L}$ de agua, para sostener de 5.75 a $11.50 \mathrm{~kg}$ de peces, que requerirán hasta $0.115 \mathrm{~kg}$ de comida al día. Estos datos se calcularon a partir de la superficie abarcada por el área de cultivo vegetal $\left(2.30 \mathrm{~m}^{2}\right)$, la cual fue estimada ocupando el modelo 3D virtual generado en el software Solidworks ${ }^{\circledR}$ 


\subsubsection{Acuario}

Además de proveer el espacio para los peces, el acuario es el contenedor que alberga el agua que circulará por el S. A. Se determinó que el material pertinente para su fabricación es el vidrio (figura 12a), ya que provee una excelente apariencia, permite que se generen superficies curvas en sus paredes y brinda una mayor facilidad de producción, en comparación con algunos materiales plásticos como el acrílico y el polietileno de baja densidad (LDPE). Su estructura está conformada por seis piezas (figura 12b) unidas mediante un adhesivo para soldado de alta resistencia activado por luz ultravioleta. Las dimensiones del acuario son de $1.50 \times 0.80 \times 0.40$ $\mathrm{m}^{3}$, y su peso es de $82.60 \mathrm{~kg}$. De acuerdo con sus características, considerando las recomendaciones brindadas por Teton (2003), el vidrio de fabricación tiene que ser de $0.01 \mathrm{~m}$ de espesor, con una densidad de $2457.60 \mathrm{~kg} / \mathrm{m}^{3}$.
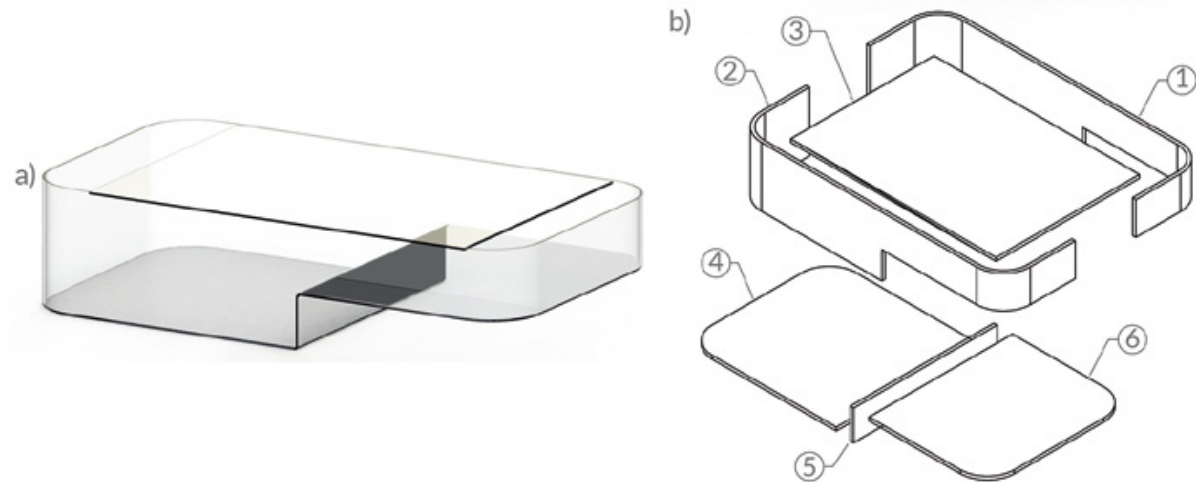

Figura 12a. Vista general del acuario; Figura 12b. Despiece del acuario. Fuente: Elaboración propia, 2019.

\subsubsection{Filtros de agua}

Como se mostrará más adelante, el equipo de investigación determinó combinar en el SA las técnicas NFT (áreas horizontales) y de cama de sustrato (áreas verticales). Esto requirió que se implementaran dos filtros, el primero ubicado entre el acuario y la bomba, y el segundo localizado entre el contenedor NFT y el acuario, antes de que el agua retorne al SA. Por sugerencia de los especialistas en hidroponía y acuaponía, el primer filtro debería captar y retener materia orgánica (comida o desechos de los peces), para evitar bloqueos en la bomba y en las tuberías. Su funcionamiento es importante, ya que los desechos no filtrados pueden crear grandes problemas debido a la descomposición de la materia orgánica. Debido a lo anterior, el primer filtro requiere de una limpieza constante. Por su parte, el segundo filtro necesita 
de una limpieza más esporádica, ya que su tarea consiste en retener algunas raíces pequeñas que logren desprenderse del área de cultivo NFT. Para el caso del primer filtro, los especialistas recomendaron implementar uno de tipo comercial en la tubería de succión de agua, de fácil instalación y limpieza, compuesto por un contenedor cilíndrico con una esponja que retiene la materia orgánica y evita que llegue a la bomba. En el segundo filtro se aconsejó utilizar una criba, compuesta por una extensión (soporte) y una malla de nylon posicionados entre el contenedor NFT y el acuario, para que por efecto de la gravedad el agua pase por él y retenga las raíces de los cultivos; este es un elemento que también resulta fácil de instalar y limpiar.

\subsubsection{Módulos de sustrato}

El equipo de investigación diseñó a su vez contenedores para cultivar individualmente cada planta (figura 13). Para su proyección se consideraron la altura que alcanzan las plantas, la profundidad que desarrollan las raíces y la separación adecuada que tiene que existir entre cada espécimen. Los contenedores de sustrato se distribuyen de manera vertical, son módulos con tres nervios radiales y dos nervios concéntricos para reforzar su estructura, pudiendo soportar cada uno hasta $4 \mathrm{~kg}$ de sustrato húmedo.
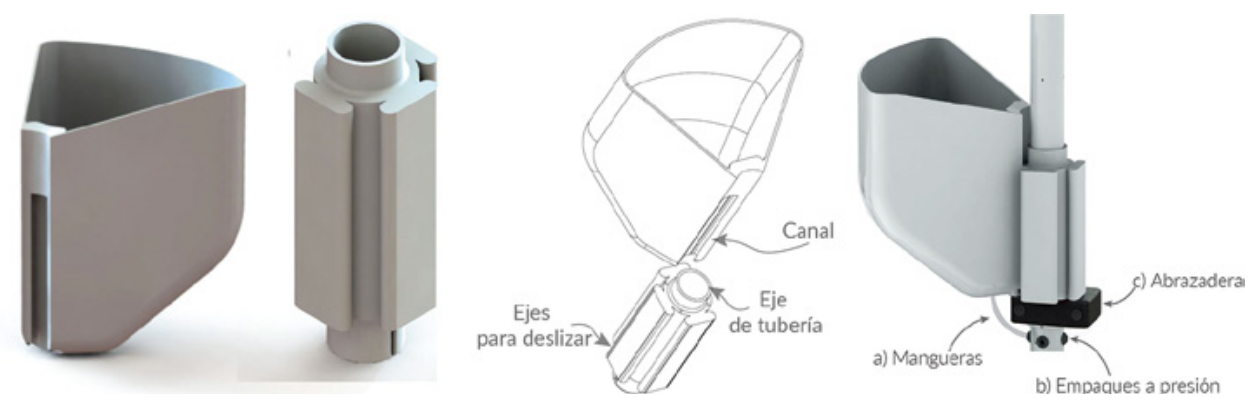

Figura 13. Esquema de ensamble de contenedores y soportes cilíndricos. Fuente: Elaboración propia, 2019. 
Los contenedores se enganchan concéntricamente a soportes cilíndricos que tienen la capacidad para albergar tres módulos mediante ranuras lineales; estos soportes cilíndricos se fijan a su vez a tubos verticales por medio de abrazaderas localizadas en su parte inferior. Los contenedores incluyen un sistema de mangueras conectadas a los tubos verticales, para reincorporar el agua sobrante al sistema de circulación. Se utilizará arlita o arcilla expandida como sustrato en los contenedores (con una densidad que oscila entre $325-750 \mathrm{~kg} / \mathrm{m}^{3}$ ), ya que se caracteriza por tener una buena capacidad de drenado y proporciona una adecuada aireación a los vegetales (Martínez y Roca, 2011). Además, se determinó para los contenedores un diámetro de $0.28 \mathrm{~m}$ y una altura de $0.25 \mathrm{~m}$, con capacidad para almacenar $3 \mathrm{~kg}$ de sustrato seco o $4 \mathrm{~kg}$ de sustrato húmedo, con un peso que oscila entre $3.55-4.55 \mathrm{~kg}$. Pueden fabricarse mediante moldeo por compresión, usando polietileno de alta densidad (HDPE) de $3 \times 10-{ }^{3} \mathrm{~m}$ de espesor. Por su parte, los soportes cilíndricos son de $0.08 \mathrm{~m}$ de largo, 0.07 $\mathrm{m}$ de ancho y $0.20 \mathrm{~m}$ de altura, con un peso propio de $0.452 \mathrm{~kg}$, y también pueden producirse a través de moldeo por compresión con HDPE de $3 \times 10-{ }^{3} \mathrm{~m}$ de espesor.

\subsubsection{Contenedor NFT}

El contenedor NFT mide $1.20 \times 0.80 \times 0.11 \mathrm{~m}$, y está conformado por tres partes principales: carcasa del contenedor, canal de riego y canastillas. Las canastillas (figura 14a) son piezas independientes hechas de polipropileno, material resistente a la humedad que evita el crecimiento de microorganismos. En ellas se colocan las plantas, y cuentan con un orificio en su parte central para que las raíces entren en contacto con la solución nutritiva que corre debajo. La carcasa del contenedor se constituye también de tres piezas: base, tapa y extensión de la base. La base (figura 14b) tiene una retícula con nervios, para evitar la flexión en su zona céntrica, y una pendiente de $2^{\circ}$ para evitar que el agua se estanque. La tapa (figura 14c) tiene una retícula similar a la de la base, con refuerzos cónicos en las paredes intermedias de los canales interiores para soportar las cargas verticales. La extensión (figura 14d) sirve para colocar una malla de nylon suave que actúa como filtro cuando el agua retorna al acuario. Las tres piezas se fabrican con HDPE.

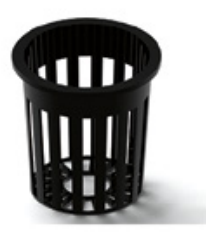

a)

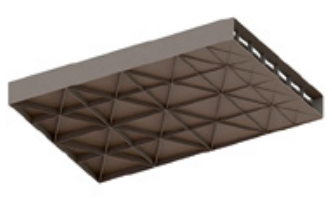

b)

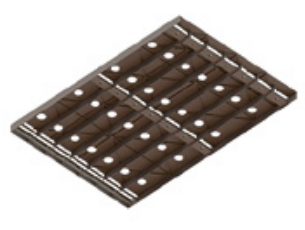

c)

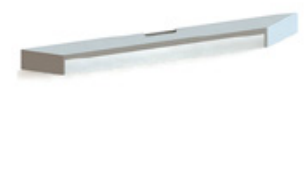

d)

Figura 14a. Canastilla NFT; Figura 14b. Base del contenedor; Figura 14c. Tapa del contenedor; Figura 14d. Extensión del contenedor. Fuente: Elaboración propia, 2019. 
El canal de riego está conformado igualmente por tres piezas fabricadas mediante inyección de HDPE: la caja de riego, la cara superior y las extremidades hidráulicas. En la cara superior (figura 15a) se ensamblan los tubos verticales de PVC con las extremidades hidráulicas, mediante soldadura en placa caliente. La caja de riego (figura 15b) presenta siete perforaciones para irrigar los contenedores. Cada contenedor contempla espacios para tres canales de riego que sirven para captar y distribuir el agua.

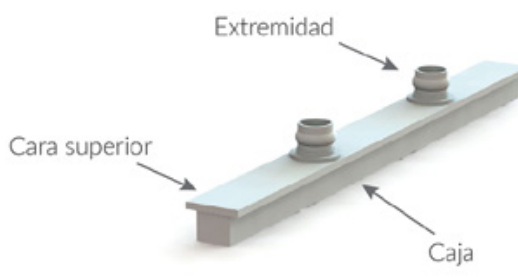

a)

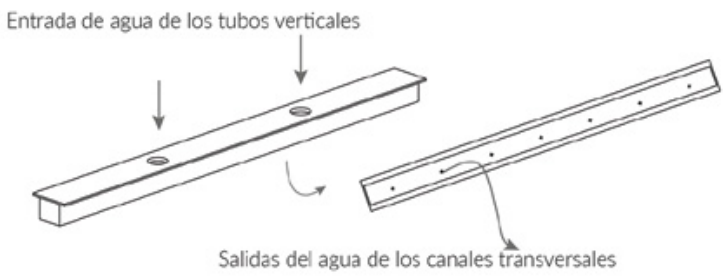

b)

Figura 15a. Ubicación de extremidades hidráulicas; Figura 15b. Ubicación de perforaciones de irrigación. Fuente: Elaboración propia, 2019.

\subsubsection{Sistema de iluminación}

Se determinó incluir un sistema de iluminación que permita a las plantas efectuar la fotosíntesis cuando el SA se ubique en un espacio interior. De acuerdo con las recomendaciones del experto en hidroponía y con las pautas expuestas por Bures, Urrestarazu y Ross (1994), Salisbury, Urrestarazu y Kotiranta (2018) y Tark (2019), se estableció que este sistema debería brindar una PAR entre los 400 y $700 \mathrm{~W} / \mathrm{m}^{2}$ y una PPFD superior a $500 \mu \mathrm{mol} / \mathrm{m}^{2} \mathrm{~s}$. Se seleccionó un panel de focos LED que ocupa un volumen de $0.31 \times 0.21 \times 0.06 \mathrm{~m} 3$, con arneses metálicos para engancharse a la estructura del SA y ajustarse a diferentes alturas; el sistema abarca un espectro completo de luz roja, azul y blanca, y brinda una PPFD que oscila entre 210 y 1712 $\mu \mathrm{mol} / \mathrm{m}^{2} \mathrm{~s}$ al colocarlo en alturas que varían de 0.77 a $0.16 \mathrm{~m}$ de la superficie de cultivo, respectivamente. El tiempo de iluminación recomendado por el fabricante, dependiendo de las especies vegetales cultivadas, varía en intervalos de 18/6 horas (encendido/apagado) o de 20/4 en la etapa vegetativa, y de 12/12 en la etapa de floración. El sistema resulta ideal para el cultivo de tomates, brócoli, lechugas, hierbas, orquídeas, entre otras plantas (Amazon, 2019), cuya distribución se define 
considerando las recomendaciones del experto en hidroponía y de Montaldo (1982), tomando en cuenta las características del sistema de iluminación y los niveles relativos de luz y sombra requeridos para cada especie vegetal, los cuales difieren conforme a su localización en el SA (figuras 16-17).

\begin{tabular}{|c|c|c|c|c|}
\hline Especie & Método de cultivo & \multicolumn{2}{|c|}{$\begin{array}{c}\text { Iluminación } \\
\text { (Directa/sombra) }\end{array}$} & Zona \\
\hline $\begin{array}{l}\text { Lechuga } \\
\text { (Lactuca sativa) }\end{array}$ & NFT & $x$ & & Zona D \\
\hline $\begin{array}{l}\text { Tomate/Jitomate } \\
\text { (Solanum lycopersicum) }\end{array}$ & Sustrato & & $x$ & Zona B \\
\hline $\begin{array}{l}\text { Fresa } \\
\text { (Fragaria) }\end{array}$ & Sustrato & & $x$ & Zona B \\
\hline $\begin{array}{l}\text { Cilantro } \\
\text { (Coriandrum sativum) }\end{array}$ & Sustrato & & $x$ & Zona B \\
\hline $\begin{array}{l}\text { Acelgas } \\
\text { (Beta vulgaris var. cicla) }\end{array}$ & $\begin{array}{c}\text { NFT } \\
\text { Sustrato }\end{array}$ & $x$ & & $\begin{array}{l}\text { Zona A } \\
\text { Zona D }\end{array}$ \\
\hline $\begin{array}{l}\text { Chile } \\
\text { (Capsicum annuum) }\end{array}$ & $\begin{array}{c}\text { NFT } \\
\text { Sustrato }\end{array}$ & & $x$ & $\begin{array}{l}\text { Zona B } \\
\text { Zona C }\end{array}$ \\
\hline $\begin{array}{l}\text { Perejil } \\
\text { (Petroselinum crispum) }\end{array}$ & Sustrato & $x$ & & Zona B \\
\hline $\begin{array}{l}\text { Orégano } \\
\text { (Origanum vulgare) }\end{array}$ & $\begin{array}{c}\text { NFT } \\
\text { Sustrato }\end{array}$ & $x$ & $x$ & $\begin{array}{l}\text { Zona A } \\
\text { Zona C }\end{array}$ \\
\hline $\begin{array}{l}\text { Hierba Buena } \\
\text { (Mentha spicata) }\end{array}$ & Sustrato & X & & Zona A \\
\hline $\begin{array}{l}\text { Espinaca } \\
\text { (Spinacia oleracea) }\end{array}$ & $\begin{array}{c}\text { NFT } \\
\text { Sustrato }\end{array}$ & & $x$ & $\begin{array}{l}\text { Zona B } \\
\text { Zona C }\end{array}$ \\
\hline
\end{tabular}

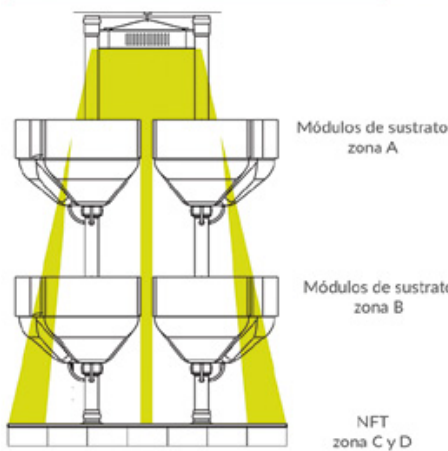

a)

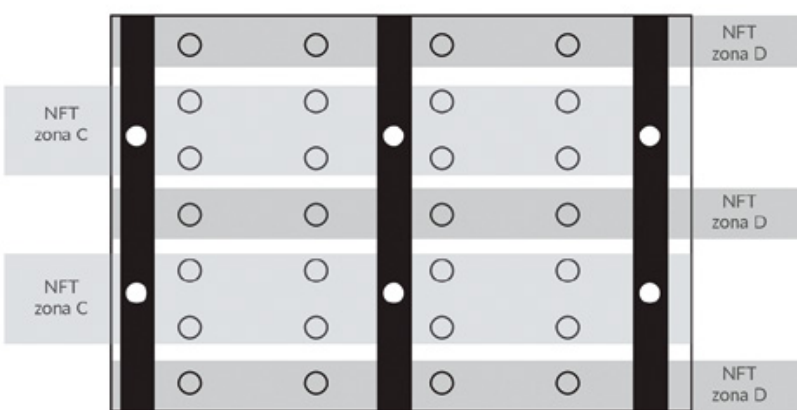

b)

Figura 16. Lista con las zonas, método de cultivo e iluminación para las especies vegetales.

Figura 17a. Distribución de plantas en los contenedores verticales; Figura 17b. Distribución horizontal en los contenedores NFT. 


\subsubsection{Estructura metálica y estante de materiales}

La estructura metálica (figura 18a) se fabricará combinando perfiles cuadrados de 0.025 y 0.038 m (1" y 1 1/2") de acero al carbono de densidad $7900 \mathrm{~kg} / \mathrm{m}^{3}$; sus dimensiones son de $1.85 \mathrm{~m}$ de largo, por $0.80 \mathrm{~m}$ de ancho, por $1.96 \mathrm{~m}$ de altura, con ruedas en su parte inferior para trasladar el SA. En la base van los perfiles metálicos de mayor tamaño, con secciones de lámina galvanizada calibre 22 para dar cuerpo a la estructura; las uniones se realizarán mediante soldadura con electrodo revestido. Por su parte, el estante de materiales (figura $18 \mathrm{~b}$ ) mide $0.37 \mathrm{~m}$ de largo, por 0.23 $\mathrm{m}$ de ancho, por $1.12 \mathrm{~m}$ de alto, y tiene cuatro entrepaños para almacenar diversos objetos. Su cuerpo está armado con tablero de fibra de densidad media (MDF) de $0.018 \mathrm{~m}$ de espesor, recubierto con melamina lisa, posee barras de protección y una agarradera de acero inoxidable. La estructura metálica se encarga de soportar al acuario, la instalación hidráulica, el sistema de iluminación, las zonas de cultivo de vegetales y el estante de materiales.

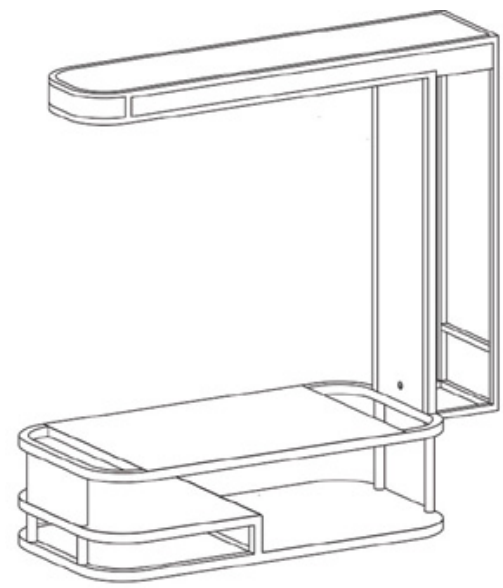

a)

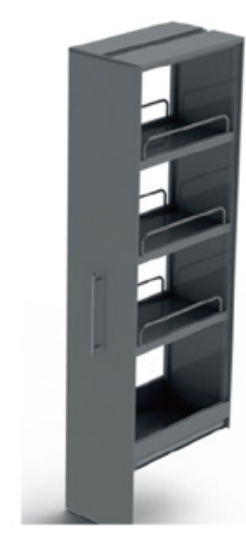

b)

Figura 18a. Estructura metálica;

Figura 18b. Estante para materiales. Fuente: Elaboración propia, 2019. 


\subsubsection{Instalación hidráulica}

Se calculó el caudal de agua que deberá impulsar la bomba, aplicando las ecuaciones de conservación de la masa, la ecuación de conservación de la energía y la ecuación de Bernoulli. Aproximadamente, la bomba deberá distribuir un caudal de $1.04 \mathrm{~L} / \mathrm{s}$; para ello se seleccionó una bomba comercial con la capacidad de impulsar un caudal de $1.19 \mathrm{~L} / \mathrm{s}$, lo cual será suficiente para garantizar que el agua circule en todo el SA. Por otro lado, para irrigar el agua se eligieron conectores y tubos de policloruro de vinilo (PVC) de $0.038 \mathrm{~m}$ (1 1 1/2") de diámetro (figura 19); además, el sistema lleva un temporizador que activará la bomba cada hora durante 14 segundos.

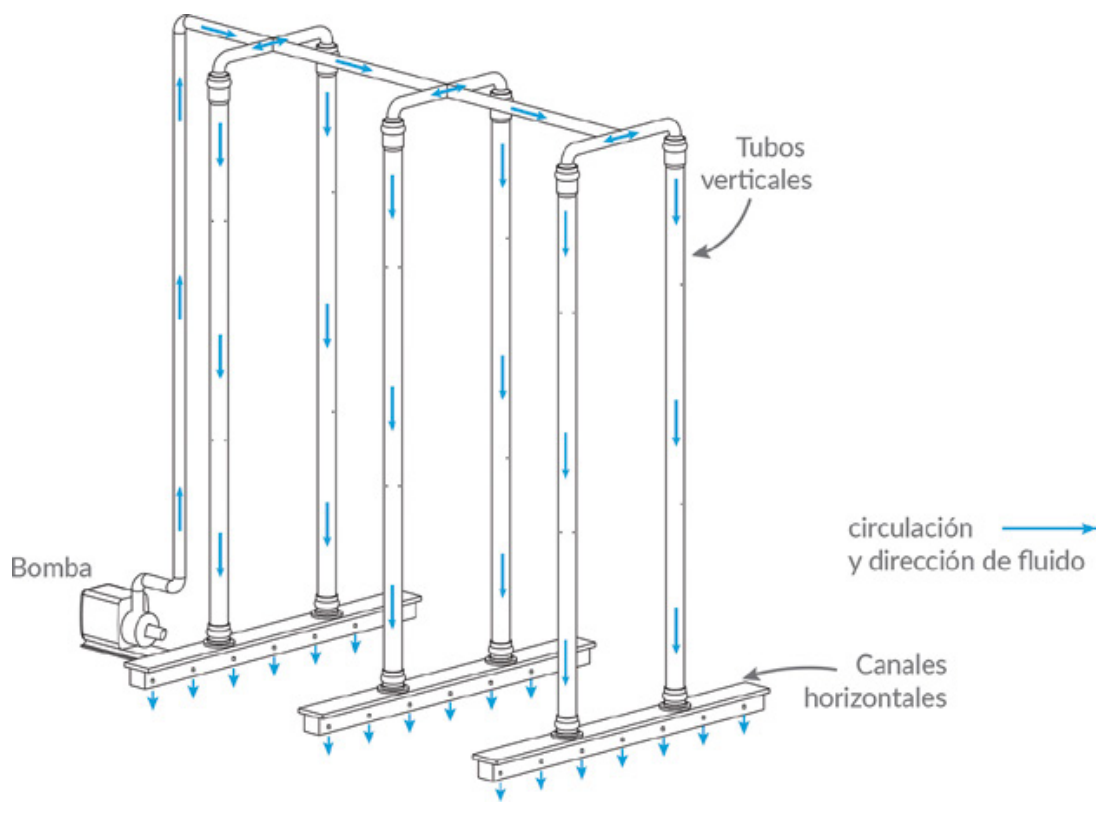

Figura 19. Esquema general de la instalación hidráulica del SA. Fuente: Elaboración propia, 2019. 


\subsection{Etapa 3. Desarrollo avanzado}

El equipo de investigación generó el modelo 3D y los planos constructivos del SA por medio del software Solidworks ${ }^{\circledR}$. De la misma forma, se efectuó una simulación mediante el análisis de elemento finito (AEF) para evaluar la resistencia de los materiales propuestos en el modelo 3D. En las simulaciones hechas con diferentes componentes, se calculó que el valor del esfuerzo de Von Mises fue menor al límite elástico de los materiales contemplados (figura 20), lo cual indica que los componentes no se romperán. Igualmente, se obtuvieron valores mayores a 1 del factor de seguridad, es decir, los elementos resistirán las cargas aplicadas.

\begin{tabular}{|l|l|l|l|l|l|}
\hline Elemento & $\begin{array}{l}\text { Carga aplicada }(\mathrm{N}, \\
\mathrm{kg})\end{array}$ & $\begin{array}{l}\text { Límite elástico del } \\
\text { material }\left(\mathrm{N} / \mathrm{m}^{2}\right)\end{array}$ & $\begin{array}{l}\text { Esfuerzo de Von } \\
\text { Mises }\left(\mathrm{N} / \mathrm{m}^{2}\right)\end{array}$ & $\begin{array}{l}\text { Factor de Seguridad } \\
\text { mínimo }\end{array}$ & $\begin{array}{l}\text { Carga máxima que } \\
\text { soportará }(\mathrm{N}, \mathrm{kg})\end{array}$ \\
\hline Contenedor de sustrato & $39.24,4$ & $2.06 \times 10^{7}$ & $3.26 \times 10^{6}$ & 6.3 & $247.21,25.20$ \\
\hline $\begin{array}{l}\text { Soporte cilíndrico del } \\
\text { contenedor de sustrato }\end{array}$ & $117.72,12$ & $2.06 \times 10^{7}$ & $1.49 \times 10^{5}$ & 140 & $1.65 \times 10^{4}, 1680$ \\
\hline Canal de riego NFT & $600.18,61.18$ & $2.06 \times 10^{7}$ & $9.85 \times 10^{5}$ & 20.92 & $1.25 \times 10^{4}, 1.28 \times 10^{3}$ \\
\hline Contenedor NFT & $1823.37,185.87$ & $2.06 \times 10^{7}$ & $1.32 \times 10^{7}$ & 1.6 & $2917.39,297.39$ \\
\hline $\begin{array}{l}\text { Área superior de la } \\
\text { estructura metálica }\end{array}$ & $177.56,18.1$ & $3.52 \times 10^{8}$ & $1.48 \times 107$ & 23.75 & $4217.05,429.87$ \\
\hline $\begin{array}{l}\text { Área inferior de la } \\
\text { estructura metálica }\end{array}$ & $8229.61,838.90$ & $3.52 \times 10^{8}$ & $1.02 \times 10^{8}$ & 3.43 & $2.82 \times 10^{4}, 2877.43$ \\
\hline
\end{tabular}

Figura 20. Resultados del AEF aplicado en el SA. Fuente: Elaboración propia, 2019. 


\section{DISCUSIÓN}

Como parte del proyecto desarrollado, el grupo de investigación hizo un análisis comparativo de los atributos del SA conceptualizado con los de un producto de referencia llamado Grove, cuyas características se aproximan a las del producto generado. Como se presenta en la figura 21, primero se compararon los datos de los espacios y áreas de cultivo, pudiendo observarse que en el SA desarrollado es posible cultivar hasta 64 plantas, cantidad que es ocho veces superior a la del sistema de referencia. Además, la superficie cultivable con vegetales es casi trece veces mayor en el producto conceptualizado, debido principalmente al aprovechamiento del espacio que se hizo distribuyendo los contenedores de sustrato NFT en diferentes niveles, tanto en sentido vertical como horizontal.

\begin{tabular}{|l|l|l|l|l|l|}
\hline Producto & $\begin{array}{l}\text { Cantidad de plantas que pueden } \\
\text { cultivarse (número de plantas) }\end{array}$ & $\begin{array}{l}\text { Área ocupada por el } \\
\text { acuario }\left(\mathrm{m}^{2}\right)\end{array}$ & $\begin{array}{l}\text { Área ocupada por los } \\
\text { vegetales }\left(\mathrm{m}^{2}\right)\end{array}$ & $\begin{array}{l}\text { Área total de } \\
\text { cultivo }\left(\mathrm{m}^{2}\right)\end{array}$ & $\begin{array}{l}\text { Área efectiva } \\
\text { ocupada }\left(\mathrm{m}^{2}\right)\end{array}$ \\
\hline Producto "Grove" & 8 & 0.32 & 0.18 & 0.5 & 0.32 \\
\hline SA proyectado & 64 & 1.2 & 2.3 & 3.5 & 1.35 \\
\hline
\end{tabular}

Figura 21. Tabla comparativa de la cantidad de espacios y áreas de cultivos. Fuente: Elaboración propia, 2019.

De igual manera, el SA diseñado ocupa un área de $1.35 \mathrm{~m}^{2}$, lo cual representa menos de la mitad del espacio que los usuarios mencionaron que podrían destinar para su implementación $\left(4 \mathrm{~m}^{2}\right)$. También, como se muestra en la figura 22, se evaluó el cumplimiento de los requerimientos de diseño establecidos en esta investigación para ambos sistemas acuapónicos mediante la primera matriz del DFC. Se utilizó una escala de Likert, para asignar un puntaje de 0 si el producto cumplió inferiormente con el requerimiento en contraste con el otro, 2.5 si cumplió de igual forma y 5 si cumplió superiormente con el requerimiento. Puede concluirse que el SA proyectado es mejor principalmente en cuatro requerimientos de diseño: 1) combina cultivos verticales y horizontales; 2) optimiza espacios; 3) permite guardar instrumentos y alimento; y 4) las áreas de cultivo están seccionadas dependiendo de las diferentes especies. 


\begin{tabular}{|c|c|c|c|c|}
\hline Requerimientos & 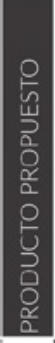 & 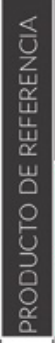 & 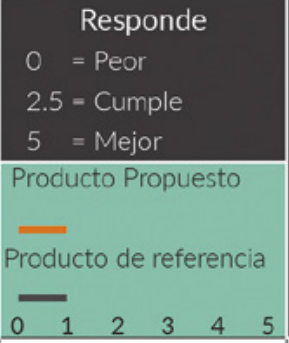 & $\frac{\frac{\pi}{u}}{\frac{1}{\tilde{u}}}$ \\
\hline EI SA es mecánicamente resistente & 5 & 4 & & 1 \\
\hline El SA combina cultivo vertical y horizontal & 5 & 0 & & 5 \\
\hline El SA optimiza espacios & 5 & 1 & & 4 \\
\hline El SA es estético & 2.5 & 2.5 & & 0 \\
\hline El SA es fácil de instalar & 2.5 & 2.5 & & 0 \\
\hline El acuario contiene la cantidad de agua adecuada al área de cultivo & 2.5 & 2.5 & & 0 \\
\hline EI SA puede cultivar 10 especies diferentes de plantas & 2.5 & 2.5 & & 0 \\
\hline El SA tiene un caudal propicio & 2.5 & 2.5 & & 0 \\
\hline EI SA provee iluminación adecuada & 2.5 & 2.5 & & 0 \\
\hline El SA es seguro para el usuario (adultos y niños) & 2.5 & 2.5 & & 0 \\
\hline EI SA ocupa un espacio máximo de $4 \mathrm{~m}^{2}$ & 3 & 2 & & 1 \\
\hline $\begin{array}{l}\text { El SA brinda aireación para oxigenar el acuario y las áreas de } \\
\text { cultivo }\end{array}$ & 2.5 & 2.5 & & 0 \\
\hline EI SA permite guardar instrumentos $y$ alimento & 4 & 1 & & 3 \\
\hline $\begin{array}{l}\text { Las áreas de cultivo están seccionadas dependiendo de las diferentes } \\
\text { especies }\end{array}$ & 5 & 0 & & 5 \\
\hline EI SA no permite el estancamiento de agua & 2.5 & 2.5 & & 0 \\
\hline EI SA filtra el agua para retener materia orgánica & 2.5 & 2.5 & & 0 \\
\hline El filtro permite su fácil limpieza & 2.5 & 2.5 & & 0 \\
\hline
\end{tabular}

Figura 22. Matriz comparativa de requerimientos entre el SA desarrollado y el de referencia. Fuente: Elaboración propia, 2019.

Adicionalmente, se aplicó una encuesta a una muestra de usuarios potenciales (72 personas), pertenecientes a los niveles socioeconómicos $\mathrm{A} / \mathrm{B}$ y $\mathrm{C}+$ de la ciudad de Huajuapan de León; en ella se les pidió que compararan ambos sistemas acuapónicos y eligieran aquel que presentara las mejores características. El 85\% de los encuestados (61 personas) seleccionó al SA conceptualizado en esta investigación como el producto de su preferencia. 


\section{CONCLUSIONES}

En esta investigación, la aplicación conjunta de la metodología propuesta por Riba y Molina (2006) y de algunas herramientas del diseño concurrente como DFC, CAD y CAE, facilitó el trabajo interdisciplinario de ingenieros en diseño, ambientales y mecánicos, además de diseñadores industriales, generando un entorno de trabajo en donde se compartieron conocimientos relacionados con mecánica de fluidos, antropometría, hidroponía y acuicultura para conceptualizar este sistema acuapónico para una producción de autoconsumo.

Mediante un análisis del entorno de mercado hecho por el equipo de investigación, se identificó la oportunidad para desarrollar un SA destinado para hombres y mujeres entre los 24 y 35 años, pertenecientes a los niveles socioeconómicos $\mathrm{A} / \mathrm{B}$ y $\mathrm{C}+$ de la ciudad de Huajuapan de León. Estas personas se caracterizan por interesarse en el cultivo de plantas y el cuidado del medio ambiente, y son individuos a los que les gustaría reducir los gastos en su alimentación generando sus propios alimentos de forma sustentable, además de que habitan en una región árida en donde existe una constante escasez de agua y se tienen pocas áreas agrícolas.

El SA se proyecta para que ocupe una superficie menor a dos metros cuadrados, y su mayor innovación radica en la integración de áreas de cultivo verticales y horizontales, lo que permite un mayor aprovechamiento de la superficie de producción. En él podrán sembrarse hasta 64 plantas de 10 diferentes especies, y cultivarse peces de 3 especies el acuario.

El producto conceptualizado posee un diseño contemporáneo de formas regulares, espesores finos, superficies lisas y colores de baja luminosidad; puede integrarse en un contexto urbano como un elemento funcional y ornamental, en áreas reducidas o en desuso, tanto en espacios exteriores como interiores.

Este SA representa una alternativa ante la escasa oferta de sistemas acuapónicos que existe en México, y en su proyección se buscó abordar las deficiencias de productos con atributos similares que actualmente se fabrican y comercializan en el país. Como trabajo futuro, se espera fabricar el prototipo físico del SA conceptualizado para probar su eficiencia como alternativa de producción agropecuaria y posteriormente obtener el registro de una patente. 


\section{REFERENCIAS BIBLIOGRÁFICAS}

Amazon (2019). Plant Grow Light, Tolys Double Switch 1000W LED Grow Lights with Timer and Thermometer Humidity Monitor, with Adjustable Rope, Full Spectrum Grow Lamps for Indoor Plants Veg and Flower (Black). Amazon [en línea, consultado en julio de 2019] Recuperado de: https:/www.amazon.ca/Thermometer-Humidity-Monitor-Adjustable-Spectrum/dp/B07MNRBP27/ ref=pd_sbs_86_1/135-0462258-4634431?_encoding=UTF8\&pd_rd_i=B07MNRBP27\&pd_rd_r=524e69d1624d-4377-98c6-58e8d93b2202\&pd_rd_w=KMJKD\&pd_rd_wg=kEL8M\&pf_rd_p=74b54c94-71954620-ba51-7d167ac58a58\&pf_rd_r=N24ANPYR3A0QA32KD04V\&psc=1\&refRID=N24ANPYR3A 0QA32KD04V

Asociación Mexicana de Agencias de Investigación y Opinión Pública-Amai (2016). Nivel Socio Económico AMAI 2018. Nota Metodológica [en línea] consultado en marzo de 2020 en: http://www.amai.org/ nse/wp-content/uploads/2018/04/Nota-Metodolo\%CC\%81gico-NSE-2018-v3.pdf

Ávila, R., Prado, L. R., y González, E. L. (2007). Dimensiones antropométricas de la población latinoamericana: México, Cuba, Colombia, Chile. México: Universidad de Guadalajara.

Bedolla, D. (2002). Diseño sensorial, las nuevas pautas para la innovación, especialización, y personalización del producto (Tesis de doctorado). Universidad Politécnica de Cataluña, España.

Bures, S., Urrestarazu, M., Ross, C. W. (1994). Fisiología vegetal. España: Grupo Editorial Iberoamérica.

Colagrosso, A. (2015). Instalación y manejo de sistemas de cultivo acuapónicos a pequeña escala (Ebook). ISBN: 9788893061988. 
Cutiño, V., Imeroni, J. C., y Sanzano, P. (2018). Acuaponía como alternativa productiva social (Tesina de licenciatura). Universidad Nacional del Centro de la Provincia de Buenos Aires, Argentina.

Díaz, I., González, C., Sención, E. y González, G. (2016). Granjas verticales: una respuesta sostenible al crecimiento urbano. Revista Tecnología y Sociedad, 7(1), 3-6.

Díaz, A., Pérez, A., y Hernández, J. (2015). Caracterización del consumidor de productos orgánicos en la ciudad de Toluca, Méx. Revista Mexicana de Agronegocios, 36, 1178-1187.

Eheim (2019). Eheim External Filters. Eheim [en línea, consultado en julio de 2019] Recuperado de: https:// www.eheim.com/en_GB/products/technology/external-filters

FAO (2003). Agro-acuicultura integrada, Manual básico. Italia: FAO.

FAO (2014). Small-scale aquaponic food production. Integrated fish and plant farming. Italia: FAO.

Gómez, F. C. et al. (2015). La acuaponía: alternativa sustentable y potencial para producción de alimentos en México. Revista Agroproductividad, 8(3), 60-65.

Gómez, M. A. (2005). Red de consumidores de productos orgánicos en la UACH: Una experiencia de organización para el consumo. Revista Vinculando. [en línea, consultado en julio de 2019] Recuperado de: http://vinculando.org/wp-content/uploads/kalins-pdf/singles/consumidores.pdf

Instituto Nacional de Estadística y Geografía-Inegi (2015). Principales resultados de la Encuesta Intercensal 2015, Oaxaca. [en línea, consultado en julio de 2019] Recuperado de: http://internet.contenidos.inegi. org.mx/contenidos/ Productos/prod_serv/contenidos/espanol/bvinegi/productos/nueva_estruc/ inter_censal/ estados2015/702825079857.pdf

Instituto Nacional para el Federalismo y el Desarrollo Municipal-Inafed (2020). Enciclopedia de los Municipios y Delegaciones de México. [en línea, consultado en enero de 2020] Recuperado de: http://www.inafed. gob.mx/work/enciclopedia/EMM20oaxaca/municipios/20039a.html

Martínez, R. (2013). La acuaponía como alternativa de producción agropecuaria sostenible, ¿Una posibilidad para tener en casa? REDICINAySA, 2(5), 16-23. 
Martínez, P. F., y Roca, D. (2011). Sustratos para el cultivo sin suelo. Materiales, propiedades y manejo. Sustratos, manejo del clima, automatización y control en sistemas de cultivo sin suelo. Colombia: Editorial de la Universidad Nacional de Colombia.

Martorell, P. (2017). El huerto vertical en casa, las plantas que mejor se adaptan. [en línea, consultado en julio de 2019] Recuperado de: https://www.vertiflor.com/blog/10-plantas-huerto-vertical/

Mondelo, P. R., Bombardo, P. B., Busquets, J. B., y Torada, E. G. (2004). Ergonomía 3: Diseño de puestos de trabajo (vol. 3). España: Universitat Politécnica de Catalunya.

Montaldo, P. (1982). Agroecología del trópico americano. Costa Rica: Instituto Interamericano de Cooperación para la Agricultura.

Pedroza, J. F., y Criado, K. (2012). Estudio de factibilidad e implementación de un cultivo acuapónico en el municipio de Ocaña, Norte de Santander (Tesis de licenciatura). Universidad Francisco de Paula Santander Ocaña, Colombia.

Ramírez, D., Sabogal, D., Jiménez, P., y Hurtado, H. (2008). La acuaponía: una alternativa orientada al desarrollo sostenible. Revista Facultad de Ciencias Básicas, 4(1), 32-51.

Ramírez, G. (2007). El mercado de productos orgánicos en Monterrey, Nuevo León y Saltillo, Coahuila (Tesis de licenciatura). Universidad Autónoma Agraria Antonio Narro, México.

Riba, C., Molina, A., (2006). Ingeniería Concurrente: Una metodología integradora. España: Edicions UPC.

Rosillón, K. et al. (2018). Sistema acuapónico vertical automatizado para la producción de alimentos (Proyecto de investigación). Universidad Privada Dr. Rafael Belloso Chacín, Venezuela.

Salisbury, F. B., Urrestarazu, M., y Kotiranta, S. (2018). Iluminación artificial en agricultura. España: Biblioteca Horticultura.

Tark, S. (2019). Todo sobre la iluminación de horticultura... Entrevista con Martin Anker, cofundador de SANlight LED. Humboldt Seed Organization. [en línea, consultado en julio de 2019] Recuperado de: https:// www.humboldtseeds.net/es/blog/iluminacion-horticultura-entrevista-martin-anker-sanlight-led/

Teton, J. (2003). Guía técnica de la acuariofilia (vol. 2). México: Ediciones AKAL.

Torres, G. (2014). Los sistemas agroalimentarios y el consumo local. México: Asociación Mexicana de Estudios Rurales. 